\title{
PLANETARY NEBULAE FROM MIRAS?
}

\author{
PATRICIA A. WHITELOCK \\ South African Astronomical Observatory, P.O. Box 9, Observatory 7935, South Africa \\ and \\ MICHAEL W. FEAST \\ Institute of Astronomy, Cambridge University, Madingley Road, Cambridge CB 3 OHA, England
}

\begin{abstract}
The properties of large amplitude AGB variables (Miras) are reviewed with particular emphasis on their relevance for evolution into PNe. We concentrate on recent observations in the LMC and Galactic Bulge. The available evidence is consistent with the idea that most Miras evolve into PNe and that most old disk stars go through the Mira and PNe phases. However, there are some suggestions that the most metal rich stars in the Galactic Bulge go through neither phase or do so very rapidly. The lifetime of a typical Bulge Mira must be more than $10^{5} \mathrm{yrs}$ and Miras outnumber $\mathrm{PNe}$ in the Bulge by about 10 to 1.
\end{abstract}

\section{Introduction}

This review deals with stars near the top of the AGB and their possible evolution into planetary nebulae. The category of highly evolved AGB stars encompasses the Miras, including those found in globular clusters, the optically invisible $\mathrm{OH} / \mathrm{IR}$ stars and their carbon-rich analogues. These are collectively referred to here as Mira Variables. Observationally we see such stars as long period $(\mathrm{P}>100$ day $)$, large amplitude $\left(\Delta M_{b o l} \gtrsim 0.5 \mathrm{mag}\right)$ pulsators. If the Mira has an optical counterpart then it will have a late spectral-type (Me, Se or Ce); otherwise it will be a strong IR source and possibly an $\mathrm{OH}$ maser. We know that these stars must be very close to the top of the AGB and we know that they have high mass-loss rates, typical values being between $10^{-7}$ and $10^{-4} M_{\odot} y r^{-1}$. Kinematic and other evidence indicates a general increase of initial mass with pulsation period from the $\sim 100$ day Miras at one end of the sequence to the $\sim 2000$ day OH/IR stars at the other.

There has been a lot of recent activity in the field of AGB evolution particularly following the results from the IRAS satellite. This review covers only a few highlights that seem particularly relevant to the relationship with PNe. In particular we concentrate on Miras in two widely different environments: the LMC and the Galactic Bulge. A more detailed review of late-type variables in the Bulge can be found in Whitelock (1992). 


\section{The LMC}

The recent results of very extensive LMC surveys of Miras have been published by, Reid et al. (1988), Feast et al. (1989), Hughes (1989) and Hughes \& Wood (1990). In addition to these major surveys some thick-shelled sources have been discovered in the LMC as a result of investigations of IRAS sources by Reid et al. (1990), Wood et al. (1991, 1992), and Reid $(1991,1992)$. Of particular interest are the $\mathrm{OH}$ sources for which Wood et al. have measured periods; the first periods for extragalactic $\mathrm{OH}$ stars. They are in excess of 1000 days and it is interesting that these $\mathrm{OH} / \mathrm{IR}$ stars fit rather well on an extrapolation of the Mira period-luminosity (PL) relation derived for Miras with periods less than 420 days (Feast et al. 1989). Given the extreme difficulty in determining the distances to OH/IR stars in the Galaxy it will be very interesting to see more observations of these and other LMC masers.

The Magellanic Clouds contain both oxygen-rich (M \& S type) and carbon-rich (C type) Miras as discussed in the above references. Of particular interest here is that the oxygen-rich Miras obey a period-luminosity-colour (PLC) relation (Feast et al. 1989). The existence of a PLC relation implies that the instability strip in the HR diagram has a finite width. Therefore some change in period of an individual Mira might be expected as it evolves through the strip. The observations however suggest rather little evolution in $\log \mathrm{P}$ at least among short period stars (see Feast \& Whitelock 1987, Whitelock 1990). A proper comparison of the PLC relation with theory requires the calculation of accurate evolutionary tracks incorporating the effects of mass loss. Although such detailed models are not available, Wood (1991) and Feast (1992) have discussed the implications of the PLC relation. Wood has suggested that the PLC relation exists because the effective temperature of the giant branch at a given luminosity varies with metal abundance. Feast has pointed out that the tracks derived by Wood imply that the short period Miras, at least, have already evolved away from the AGB due to the depletion of their envelopes by mass loss. In other words, Miras may already be post-AGB stars.

\section{The Bulge}

We (Glass, Whitelock, Feast \& Catchpole in preparation) are in the process of analyzing multi-phase observations of the Mira variables in the Sgr I Baade window $\left(l=1^{\circ} .4, b=\right.$ $\left.-2^{\circ} .6\right)$. The analysis is still in a preliminary stage but suggests that these Bulge Miras diverge somewhat from the LMC Mira PL and period-colour (PC) relations. Figure 1 shows the PC relation for Miras in the Bulge and the LMC. A few Bulge stars with extreme colours, $(\mathrm{J}-\mathrm{K})_{\mathrm{o}} \gtrsim 4$, have been omitted from this plot. Such extreme colours are found for IRAS Miras and are the product of circumstellar reddening in stars with thick dust shells (Whitelock et al. 1991). It is clear from Fig 1 that the longer period Bulge sources have distinctly redder colours than would be predicted from the LMC PC relation.

The PL relation is difficult to derive directly in the Bulge because of the finite distance spread in the line of sight. However if the LMC PL relation is compared with the measured luminosity, distance moduli can be derived for individual Miras. The distribution of distance moduli can then be compared with that predicted from a model of the Bulge and the distance to the Galactic Centre $\left(R_{o}\right)$ derived. This approach results in $R_{o}=9.1 \mathrm{kpc}$ if the 


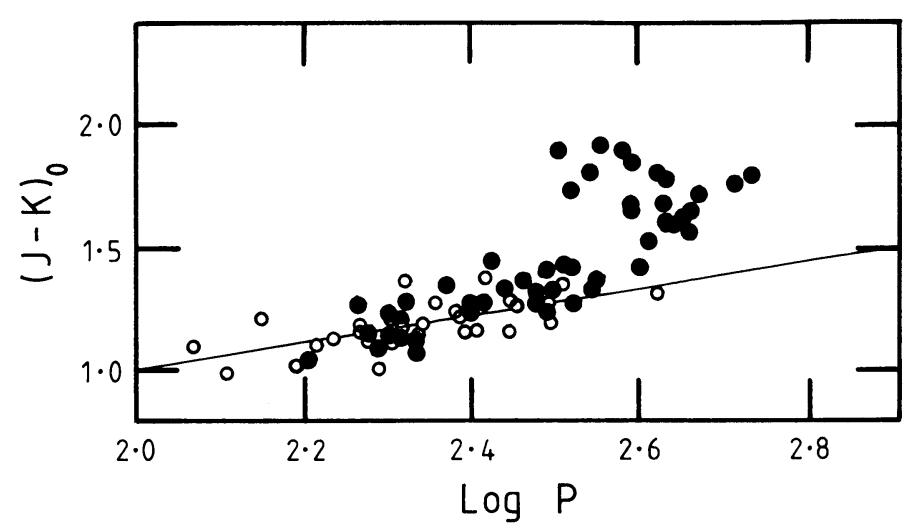

Figure 1. The PC relation for Miras in the LMC (open circles) and the Bulge (closed circles). The solid line is a least-squares fit to the LMC data.

bolometric PL relation is used, or $\mathrm{R}_{\mathrm{o}}=8.6 \mathrm{kpc}$ if the $\mathrm{K}(2.2 \mu \mathrm{m}) \mathrm{PL}$ relation is used (omitting the few sources with optically thick shells). That is, the PL relations do not give consistent results and they both imply a rather large distance to the centre (Feast 1987 gives $\mathrm{R}_{\mathrm{o}}=7.8 \pm 0.8 \mathrm{kpc}$ ). In other words the stars are fainter than the LMC PL relations predict. If instead of using the LMC PL relation to calculate the distances we use the LMC PLC relation, then the derived distance is $R_{o}=8.2 \mathrm{kpc}$ from both the $\mathrm{K}$ and the bolometric PLC relations. Figure 2 illustrates the model fit for distances derived from the bolometric PLC relation. This result confirms the existence of the PLC relation and implies that the Bulge Miras, or at least the longer period ones, occupy a slightly different part of the instability strip from the LMC Miras. Wood (1990) has suggested on theoretical grounds that we might expect metal rich Miras to behave in this way.

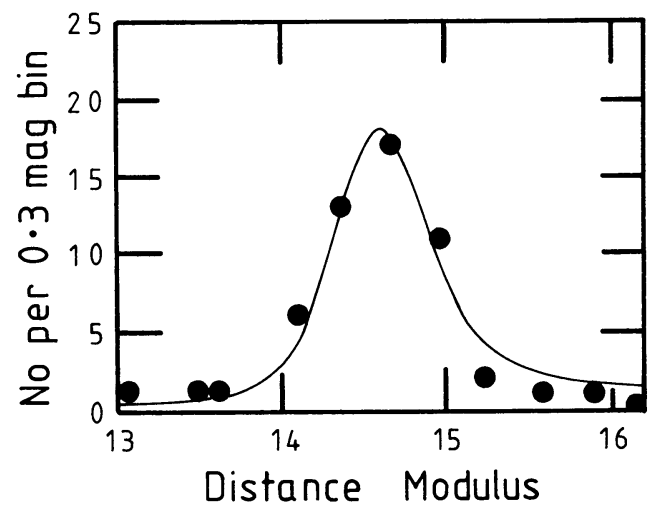

Figure 2. The distribution of distances for Miras in the Sgr I window. The observations are compared to a model with $\mathrm{R}_{\mathrm{o}}=8.2 \mathrm{kpc}$, a volume density distribution $\rho \propto a^{-2}$ and $c / a=0.6$. The standard error of a single distance modulus is assumed to be $0.2 \mathrm{mag}$. 
Whitelock et al. (1991) did a survey of Miras with high mass-loss rates in the Galactic Bulge. They colour selected IRAS sources from two strips across the Bulge between $b=$ $+7^{\circ}$ and $+8^{\circ}$ and $b=-7^{\circ}$ and $-8^{\circ}$ with $345^{\circ}<l<15^{\circ}$. They monitored these for several years in the near-infrared finding 113 Miras and deriving periods for 104 of them. Bolometric luminosities were then derived from the PL relation and apparent luminosities were calculated by integrating the flux from the IRAS and near-infrared photometry. Hence distances to individual Miras were derived. Note that the PLC relation cannot be used to derive the luminosities of and hence distances to these stars because use of the PLC relation requires the photospheric colour which is unknown for these thick shelled sources. An estimate of the error introduced by use of the PL relation rather than the PLC relation may be made from the fact that $R_{o}=8.6 \mathrm{kpc}$ is derived from use of the PL for these sources in comparison to the currently favoured value of $R_{o}=7.8 \mathrm{kpc}$. The effect of this will be noted below where appropriate.

Whitelock et al. (1991) used a slightly modified version of the expression derived by Jura (1987) to estimate the mass-loss rates $(\dot{M})$ for these Miras. The circles in Fig 3 show the derived mass-loss rates as a function of pulsation period and amplitude. The use of the PL relation to derive the distances will result in an overestimate of the mass-loss rate by about $20 \%$. Sources with periods over 600 days are shown as filled circles on the period $/ \dot{M}$ diagram; sources with amplitudes over $1.8 \mathrm{mag}$ are similarly indicated on the $\Delta \mathrm{K} / \dot{M}$ diagram. It is clear that at a given period there is a range of mass-loss rates and that the stars with the largest mass-loss rates have the largest pulsation amplitudes. This illustrates the now well known and presumably causal connection between pulsation and mass loss (see also Wood's paper in these proceedings).

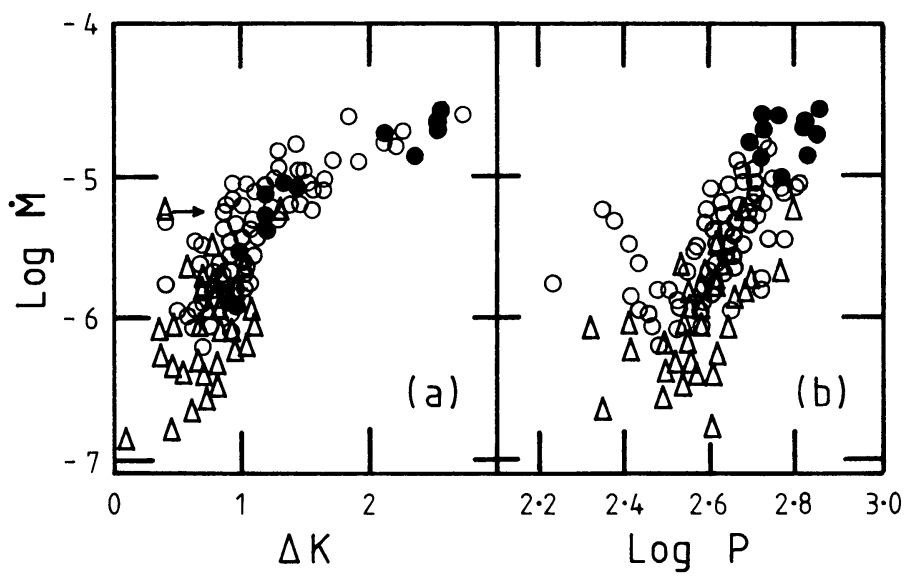

Figure 3. Mass-loss rates $(\dot{M})$ as a function of (a) pulsation amplitude and (b) period. Circles are from Whitelock et al. (1991) and triangles are from the extension of the survey as described in the text.

The Bulge survey was recently extended in the 3.8 square degree area where the $-7^{\circ}$ to $-8^{\circ}$ strip overlaps the Groningen field number 3 , near to $l=0^{\circ}$. The intention was to identify all of the Miras in this one area. JHKL photometry was obtained for all of 
the $12 \mu \mathrm{m}$ IRAS sources in this region other than the 7 already examined as part of the original survey. From the 89 stars examined 47 have the colours of Miras and periods were determined for 40 of these. The periods of these 40 covered roughly the same range as those with the extreme colours but with a somewhat lower average period. From blue plates Plaut (1971) and Wesselink (1987) found a total of 45 Miras in the overlap region, most of which have periods less than 300 days. Only 4 of these are in common with the 47 in the IRAS detected sample, suggesting that there are other Miras that were detected neither by IRAS nor on the blue plates and that the survey is therefore not complete.

Mass-loss rates were calculated for the Miras with IRAS fluxes in the same way as those described above and are plotted as triangles in Fig 3. For those with only upper limits for the $25 \mu \mathrm{m}$ flux a value of 0.56 times the $12 \mu \mathrm{m}$ flux was used to estimate $\dot{M}$, this being the average value for those which did have $25 \mu \mathrm{m}$ fluxes. It may be that the mass-loss rates for these stars are overestimated with respect to the red group in that the expansion velocity of $15 \mathrm{~km} \mathrm{~s}^{-1}$ which is assumed for all the sources is probably an overestimate for those with thinner dust shells. Nevertheless these Miras extend the sequence shown by the red group; they exhibit lower mass-loss rates and lower pulsation amplitudes over the same period range.

There were a total of 95 Miras in the 3.8 square degrees at $b=-7^{\circ} .5$ providing a lower limit for the total number of Miras per unit area at this latitude. An estimate is also available for the total number of Miras in two other inner Bulge fields, Sgr I and NGC 6522, from the work of Lloyd Evans (1976) and Glass (1986). Both of these are probably less than complete but are unlikely to be more so than the $-7^{\circ} .5$ field. Figure 4 is modified from Feast et al. (1992) and shows the distribution of Miras from the three Bulge fields as a function of galactic latitude compared to that of late-M stars (from Blanco 1988). The Mira fall off is more gradual than that of the $M$ stars; the surface density of Miras changes as $R^{-2}$ (where $\mathrm{R}$ is distance from the galactic centre) in contrast to a dependency of $\mathrm{R}^{-3.2}$ (corresponding to a volume density of $\mathrm{R}^{-4.2}$ ) found by Blanco and Terndrup (1989) for stars later than M5.

$|b|$

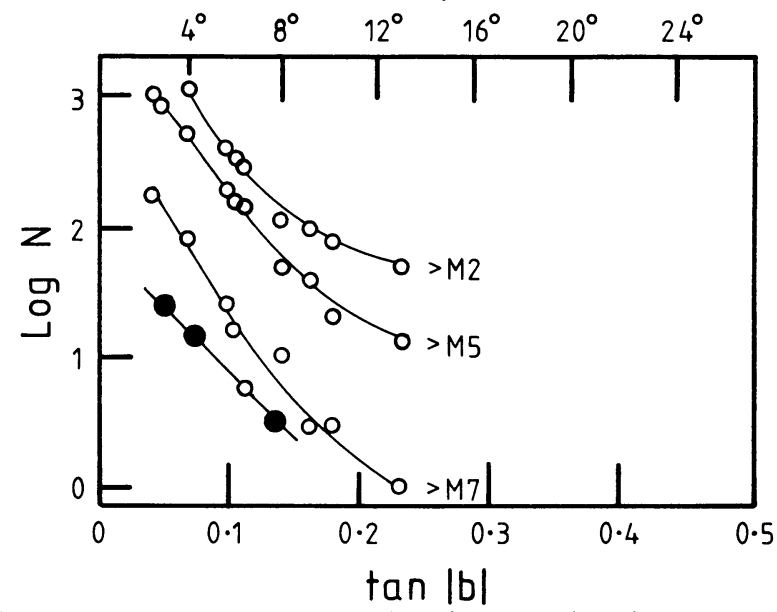

Figure 4. The galactic latitude dependence of surface number density for late-M stars (open circles) and Miras (closed circles). 
This result must be regarded as tentative until deep surveys of Miras in a number of fields in the Bulge are obtained. The $M$ stars whether they are on the giant branch or asymptotic giant branch are generally thought to be the progenitors of the Miras so this difference in distribution is rather surprising. It implies that either the most metal-rich $M$ stars do not become Miras (perhaps they become AGB-manqué stars, the existence of which has been postulated to explain the uv-excess measure for extreme metal-rich ellipticals, e.g. Renzini \& Greggio (1990)) or that the Mira descendants of metal-rich M giants may exist but have lifetimes much shorter than their globular cluster counterparts. An alternative possibility is that the late M-giants evolve predominantly into long-period Miras and that these have a much steeper density gradient than do short period ones.

\section{Mira Lifetimes}

As stated above, the $-7^{\circ} .5$ field contains 95 Miras in 3.8 square degrees, 7 of which are high mass-loss objects observed in the first survey. The total number of Miras we would expect in the two strips covered by the original survey which contains 113 high mass-loss Miras, is therefore $(113 / 7) \times 95=1534$. Approximately $13 \%$ of these will be foreground objects reducing the number to 1300 . In order to estimate the total number of Miras in the Bulge it is necessary to assume a model for the Mira distribution. The model fit to Fig 2 suggests a volume density distribution $\rho \propto \mathrm{R}^{-2}$, while Fig 4 which shows that the surface density distribution goes as $\mathrm{R}^{-2}$ would imply $\rho \propto \mathrm{R}^{-3}$. This difference is probably the result of the bar-like structure of the Bulge (Whitelock \& Catchpole 1992 and references therein) which should be explicitly modelled to determine the number of Miras. In fact our current knowledge is inadequate to do this effectively and so for the purpose of making an estimate we assume an ellipsoidal Bulge with a volume density distribution $\rho \propto \mathrm{R}^{-2}$. The total number of Miras within a radius of $2 \mathrm{kpc}$ from the centre is then at least $2 \times 10^{4}$. The effect of increasing the exponent for the volume density distribution will be to increase the estimate of the total number of Miras in the Bulge.

It is then possible to use the fuel consumption theorem from Renzini \& Buzzoni (1986) to estimate the lifetime of a typical Bulge Mira. This expression:

$$
t_{j}=N_{j} / B(t) L_{T}
$$

relates the duration of a specific evolutionary phase, $t_{j}$, to the number of objects in that phase, $N_{j}$, via the total luminosity of the population, $L_{T}$, and the stellar death rate per unit luminosity, $B(t)$. A lifetime of at least $10^{5} \mathrm{yrs}$ is derived. This can be compared with other estimates. Renzini and Greggio (1990) using the same expression find $2.5 \times 10^{5}$ yrs for the Globular Cluster Miras. The cluster Miras are of short period ( $<300$ days). Jura and Kleinmann (1992) have estimated a lifetime of $2 \times 10^{5}$ for Miras with intermediate periods (300-400 days). The Miras in the Bulge cover a very large period range from 100 to perhaps 800 days, but the bulk of them will have periods less than 400 days.

The numbers of Miras can be compared with the numbers of PNe. Pottasch (1992) has estimated that there are at least $1000 \mathrm{PNe}$ within $10^{\circ}$ of the centre. An estimate for Miras within the same radius is $1.4 \times 10^{4}$ giving a ratio of Miras to PNe of 14 . The only Bulge field surveyed in depth for both PNe and Miras is the Baade window near NGC 6522, which contains 114 Miras per square degree and 9 PNe per square degree (from Shaw \& 
Wirth (1985) and Acker, private communication) giving a ratio of 13. Pottasch estimates the observable lifetimes of Bulge PNe as 8000 yrs compared to our estimate of $>10^{5} \mathrm{yrs}$ for Miras; this gives a ratio of $>12$. All these are in remarkably good agreement given the uncertainties and are consistent with all Bulge PNe evolving from Miras even if not all $\mathrm{M}$ giants become Miras.

The view that all or most Miras evolve into $\mathrm{PNe}$ is also consistent with other evidence. PNe evolve from stars with a range of initial masses, but the bulk of PNe appear to have evolved from low mass stars. This is true for PNe both in the Galactic Bulge (where there is an absence of young stars) and in the extended solar neighbourhood where the kinematics of PNe shows that the bulk of the objects must belong to an old disk population (radial velocity dispersion $\sim 40 \mathrm{~km} \mathrm{~s}^{-1}$ and asymmetric drift $\sim 20 \mathrm{~km} \mathrm{~s}^{-1}$, cf Oort (1965), Pottasch (1983)). In view of this it has been puzzling that estimates of the scale heights of PNe in the Galaxy have given relatively small values, $100-150 \mathrm{pc}$, compared with the scale height of the old disc population ( $\sim 300-350 \mathrm{pc}, c f$ Freeman 1987$)$. However, the scale height estimates depend critically on the very uncertain distances to the PNe. Recently Zijlstra \& Pottasch (1991) have estimated a scale height of $250 \pm 50 \mathrm{pc}$ for PNe by a method which seems likely to be less dependent on the adopted distance scale. This result coupled with the discussion at this meeting by Peimbert (his table relating birthrate to scale height) would suggest that all old disk stars pass through a PN phase and that the PN birthrate per unit luminosity $(\dot{\xi})$ in the galactic disk is $\sim 20 \times 10^{-12} y r^{-1} L_{\odot}^{-1}$ consistent with the value found in other galaxies by Peimbert and with the value of the stellar death rate predicted by Renzini \& Buzzoni (1986). The decrease in $\dot{\xi}$ discussed by Peimbert for galaxies brighter or redder than our own suggests that these galaxies contain a significant population which does not evolve through the PN phase. One possibility would be that they contain an old supermetal-rich population and that these stars do not become $\mathrm{PNe}$ (or that super-metal-rich PNe have short lifetimes). This would be consistent with the results of Ratag et al. (1992) and of Clegg (these proceedings) which show that the PNe in the Galactic Bulge evolve predominantly from the relatively metal poor part of the Bulge population.

It would evidently be important to study the relative numbers and distributions of $\mathrm{PNe}$, Miras and giant stars in the Galactic Bulge as a function of metallicity (which in the case of Miras seems related to period). Such a study would place our understanding of the relation of Miras to PNe on a much firmer footing.

\section{Acknowledgements}

We are grateful to Ian Glass and Robin Catchpole for permission to quote results from work in preparation. MWF acknowledges financial support from the Royal Society.

\section{References}

Blanco, V. M. (1988) AJ, 95, 1400.

Blanco, V. M. \& Terndrup, D. M. (1989) AJ, 98, 843.

Feast, M. W. (1987) In: The Galaxy, p1, Eds. G. Gilmore \& B. Carswell, Reidel, Dordrecht. Feast, M. W. (1992) In: Highlights of Astronomy, in press, Ed. J. Bergeron, Kluwer, Dordrecht. 
Feast, M. W. \& Whitelock, P. A. (1987) In: Late Stages of Stellar Evolution, p33, Eds. S. Kwok \& S. R. Pottasch, Reidel, Dordrecht.

Feast, M. W., Glass, I. S., Whitelock, P. A. \& Catchpole, R. M. (1989) MNRAS, 241, 375. Feast, M. W., Whitelock, P. A. \& Sharples, R. (1992) In: The Stellar Populations of Galaxies, IAU Sym 149, p77, Eds. B. Barbuy \& A. Renzini, Kluwer, Dordrecht.

Freeman, K. C. (1987) Ann Rev A E⿱ $A, \mathbf{2 5}, 603$.

Glass, I. S. (1986) MNRAS, 221, 879.

Hughes, S. (1989) $A J, \mathbf{9 7}, 1634$.

Hughes, S. \& Wood, P. R. (1990) AJ, 99, 784.

Jura, M. (1987) ApJ, 313, 743.

Jura, M. \& Kleinmann, S. G. (1992) ApJ Sup, 79, 105.

Lloyd Evans, T. (1976) MNRAS, 174, 169.

Oort, J. H. (1965) In: Galactic Structure, p455, Eds. A. Blaauw \& M. Schmidt, Univ of Chicago Press.

Plaut, L. (1971) A \& A Sup, 4, 75.

Pottasch, S. R. (1992) preprint.

Pottasch, S. R. (1984) Planetary Nebulae, Reidel, Dordrecht.

Ratag, M. A., Pottasch, S. R., Dennefeld, M., Menzies, J. W. (1992) A \& A, 255, 255.

Renzini, A. \& Buzzoni, A. (1986) In: Spectral Evolution of Galaxies, p195, Eds. C. Chiosi \& A. Renzini, Reidel, Dordrecht.

Renzini, A. \& Greggio, L., (1990) In: Bulges of Galaxies, p47, Eds. B. J. Jarvis \& D. M. Terndrup, ESO Conf. \& Workshop Proc. 35.

Reid, N., Glass, I. S. \& Catchpole, R. M. (1988) MNRAS, 232, 53.

Reid, N., Tinney, C. \& Mould, J. (1990) ApJ, 348, 98.

Reid, N. (1991) ApJ, 382, 143.

Reid, N. (1992) these proceedings.

Shaw, R. A. \& Wirth, A. (1985) PASP, 97, 1071.

Wesselink, Th. (1987) Ph D Thesis, Nijmegen.

Whitelock, P. A. (1990) In: Confrontation Between Stellar Pulsation and evolution, ASP conf ser 11, p365, Eds. C. Cacciari \& G. Clementini.

Whitelock, P. A. (1992) In: Galactic Bulges, IAU sym 153, in press, Kluwer, Dordrecht.

Whitelock, P. A. \& Catchpole, R. M. (1992) In: The Center, Bulge and Disk of the Milky Way, in press, Ed. L. Blitz, Kluwer, Dordrecht.

Whitelock, P. A., Feast, M. W. \& Catchpole, R. M. (1991) MNRAS, 248, 276.

Wood, P. R. (1990) In: From Miras to Planetary Nebulae, p67, Eds. M. O. Mennessier \& A. Omont, Editions Frontiers.

Wood, P. R., Moore, G. K. G. \& Hughes, S. M. G. (1991) In: The Magellanic Clouds, IAU Sym 148, p259. Eds. R. Haynes \& D. Milne, Kluwer, Dordrecht.

Wood, P. R., Whiteoak, J. B., Hughes, S. M. G., Bessell, M. S., Gardner, F. F. \& Hyland A. R. (1992) preprint.

Zijlstra, A. A. \& Pottasch, S. R. (1991) A \& A, 243, 478. 